\title{
Modification of conductive polyaniline with carbon nanomaterials
}

\author{
Sajjad Sedaghat $\cdot$ Mahdi Soleimani Alavijeh
}

Received: 22 November 2013/ Accepted: 5 July 2014/Published online: 15 August 2014

(C) The Author(s) 2014. This article is published with open access at Springerlink.com

\begin{abstract}
The synthesis of polyaniline/single-wall nanotube, polyaniline/multi-wall nanotube and polyaniline/single-wall nanotube/graphen nanosheets nanocomposites by in situ polymerization are reported in this study. The substrates were treated with a mixture of concentrated sulfuric acid and concentrated nitric acid before usage to functionalize with carboxylic and hydroxyl groups. Aniline monomers are adsorbed and polymerized on the surface of these fillers. Structural analysis using scanning electron microscopy showed that nanomaterials dispersed into polymer matrix and made tubular structures with diameters several tens to hundreds nanometers depending on the polyaniline content. These nanocomposites can be used for production of excellent electrode materials applications in high-performance supercapacitors.
\end{abstract}

Keywords Nanocomposites $\cdot$ Polyaniline $\cdot$ Carbon nanomaterials $\cdot$ Conductive polymer $\cdot$ Supercapacitor

\section{Introduction}

Supercapacitors over the last years have attracted attentions because they could store and deliver energy at relatively high rates [1], also they have power density, cycle life, energy density [2], wide thermal operating range, and low maintenance cost better than conventional electrical double-layer

\section{S. Sedaghat $(\bowtie)$}

Department of Chemistry, College of Science Malard Branch, Islamic Azad University, Malard, Iran

e-mail: sajjadsedaghat@yahoo.com

M. S. Alavijeh

Department of Chemistry, College of Science Rasht Branch, Islamic Azad University, Rasht, Iran capacitors [3]. So far, super capacitors used are mixed with metal oxides [4] and conducting polymers [5] as electrode materials. Metal oxides because of the high cost and toxicity to environment have greatly limited its practical applications. In recent years, more researches were focused on the conducting polymers due to low materials cost and low toxicity [6]. Conducting polymers such as polyacetylene [7] polyaniline (PANI), polypyrrole and polythiophene applied for this purpose [3]. Among these polymers PANI have more notability because of its very facile synthesis, low cost and better properties and excellent environmental stability compared to others conducting polymers. [8, 9]. It has been determined that carbon nano materials such as multi-wall nanotube (MWNT), single-wall nanotube (SWNT) and graphennano sheets (GNS) could be used as famous nano fillers into PANI for improving mechanical and electrical properties $[6,9,10]$. MWNTs have been identified in 1991 and SWNTs in 1993. Carbon nanotubes (CNTs) are repeating hexagonal network of $\mathrm{sp}^{2}$ hybridized carbons [11]. The properties of these nanocomposites such as tensile strength, tensile modulus, toughness, glass transition temperature, thermal conductivity, electrical conductivity, solvent resistance and optical properties have been improved compare to pure polymers [12]. CNTs could functionalize and dispersed in the polymer matrix with carboxylic acid groups [11]. For this purpose usually treated with a concentrated mixture of nitric and sulfuric acids [13-17]. Then these oxygen groups can interleaf with monomers in the process of in situ polymerization and also prevent the agglomeration of CNTs. Another carbon material used in this work is GNS. GNS is 2-dimensional layer of carbon atoms with $\mathrm{sp}^{2}$ hybridization and one atom thickness and is prepared in 2004 [18]. It is very suitable for using in capacitors due to its excellent characteristics inclusive large surface area, chemical stability and high electrical 
conductivity and good mechanical properties compared with CNTs [1, 3]. GNS could be prepared of both chemical (reduction with hydrazine hydrate) [19] and physical (thermal shock) [20] treatments of exfoliated graphite oxide. Weak flexibility of PANI limits it from usage in high-performance flexible super capacitors. It has been modified with GNS that can enhance the electric conductivity and the mechanical strength of PANI composites [21]. Similar to CNTs aniline monomers can be adsorbed on GNS and then polymerization can begin in the surface. In this paper we have described the preparation of GNS by chemical reduction and evaluation of their morphology. In addition, we have treated SWNTs and MWNTs with sulfuric and nitric acid. Therefore, we report a simple process to synthesis composites of SWNT, MWNT and GNS which are doped with PANI by in situ polymerization. The morphology of nanocomposites has been determined using scanning electron microscopy (SEM).

\section{Experimental}

\section{Materials}

Carbon nanotubes (MWCNTs and SWNTs) were purchased from Neunano. The diameters of the commercial CNTs (purity $>95 \%$ ) are around $20-30 \mathrm{~nm}$ and the lengths are up to $5-15 \mathrm{~mm}$. GNS were prepared by reduction of graphite oxide (GO) with hydrazine hydrate [19]. Aniline monomer was purchased from Merk and was distilled twice under reduced pressure and stored below $4{ }^{\circ} \mathrm{C}$. The other reagents, such as ammonium peroxydisulfate $\left[\left(\mathrm{NH}_{4}\right)_{2-}\right.$ $\mathrm{S}_{2} \mathrm{O}_{8}$ ) ] (APS), hydrochloric acid ( $\left.\mathrm{HCl}\right), \mathrm{H}_{2} \mathrm{SO}_{4}, \mathrm{HNO}_{3}$, $\mathrm{H}_{2} \mathrm{O}_{2}$, hydrazine hydrate, acetone and ethanol were analytical grade and used without further purification.

\section{Synthesis of PANI/SWNT and PANI/MWNT nanocomposites}

$0.15 \mathrm{~g}$ of SWNTs and $0.15 \mathrm{~g}$ of MWNTs were separately refluxed in a mixture $(3: 1, \mathrm{v} / \mathrm{v})$ of concentrated sulfuric $(98$ $\mathrm{wt} \%)$ and nitric acids $(65 \mathrm{wt} \%)$ in $70{ }^{\circ} \mathrm{C}$ for $3 \mathrm{~h}$ to achieve functionalization. Then dried in an oven at $100{ }^{\circ} \mathrm{C}$ for $48 \mathrm{~h}$, then were added to $135 \mathrm{ml}$ distillate water and sonicated for $1 \mathrm{~h}$. Then, $0.25 \mathrm{M}$ aniline monomers solution $(1 \mathrm{ml}$, monomer plus $40 \mathrm{ml}$, solvent: $1 \mathrm{M} \mathrm{HCl}$ ) were added into their suspension and sonicated for $1 \mathrm{~h}$. These suspensions were kept in an ice bath at $0-5{ }^{\circ} \mathrm{C}$ and stirred extremely. Afterwards, an equal volume of $0.25 \mathrm{M}$ (APS) solution were dropped into the above mixtures and kept at $0-5{ }^{\circ} \mathrm{C}$ for $4 \mathrm{~h}$ under hard stirring and continued for $24 \mathrm{~h}$ at room temperature. Finally, SWNT/PANI and MWNT/PANI composites were filtered and washed with a large amount of distilled water and ethanol for ten times until the solution of filtrate were transparent. The powder were dried in an oven at $85{ }^{\circ} \mathrm{C}$ for $24 \mathrm{~h}$ and kept in the desiccators for 2 days. In these nanocomposites the mass ratio of PANI to CNTs was 98.5:1.5. For comparison, pure PANI was also prepared through the above-mentioned chemical process.

\section{Synthesis of PANI/GNS/SWNT nanocomposite}

\section{Preparation of GNS from GO via chemical reduction} with hydrazine hydrate

In a typical procedure, $0.1 \mathrm{~g}$ of $\mathrm{GO}$ were added on $100 \mathrm{ml}$ of distilled water and sonicated for $1 \mathrm{~h}$, then $1 \mathrm{ml}$ of hydrazine hydrate as reductant, was added into this suspension and heated in an oil bath at $100{ }^{\circ} \mathrm{C}$ under a watercooled condenser for $24 \mathrm{~h}$. The residue GO dried and keep in an oven at $100{ }^{\circ} \mathrm{C}$ for $12 \mathrm{~h}$ and $0.25 \mathrm{M}$ aniline monomers solution $(1 \mathrm{ml}$, monomer plus $40 \mathrm{ml}$, solvent: $1 \mathrm{M}$ $\mathrm{HCl})$ poured and sonicated for $1 \mathrm{~h}$ until the production suspension of GNS. This suspension kept in the bath-ice at $0-5{ }^{\circ} \mathrm{C}$ for next step.

\section{Synthesis of PANI/GNS/SWNT nanocomposite}

For this purpose, $0.05 \mathrm{~g}$ of SWNT were functionalized similar to previous section and added into $0.25 \mathrm{M}$ aniline monomers solution $(1 \mathrm{ml}$, monomer plus $40 \mathrm{ml}$, solvent: $1 \mathrm{M} \mathrm{HCl}$ ) and $0.1 \mathrm{~g} \mathrm{GNS}$ was inserted and sonicated for $1 \mathrm{~h}$ until to produce an homogeneous yellow-brown suspension. The mixture kept in an ice bath and $2.5 \mathrm{~g}$ of APS in $40 \mathrm{ml}$ of a $1 \mathrm{M} \mathrm{HCl}$ solution was dropped and stirred. Stirring continued for $4 \mathrm{~h}$ at $0-5{ }^{\circ} \mathrm{C}$ and $24 \mathrm{~h}$ at ambient temperature. Then filtered, washed with distilled water and ethanol repeatedly and dried in an oven at $85{ }^{\circ} \mathrm{C}$ for $24 \mathrm{~h}$ and this composite kept in the desiccators for 2 days. In this composite mass ratio of GNS to SWNT was 75:25.

\section{Characterization}

Scanning electron microscopy (SEM; model philips) was used to observe the morphologies of the samples. Before the SEM examination, the samples were coated with a very thin layer of gold.

\section{Results and discussion}

\section{Characterization of PANI/SWNT and PANI/MWNT composites}

When oxidizing agent was added in the mixture, polymerization began in the surface of CNTs between aniline 
monomers and resumed in the environment and oxidative polymerization occurred. Decreasing the temperature made suitable condition for oxidation of aniline. Figure 1 shows the SEM image of SWNT/PANI and Fig. 2 shows the SEM image of MWNT/PANI.

In these pictures, sunny zones are relevant to PANI and dark zones pertain to CNTs. We perceive that CNTs dispersed in the polymer matrix very well and PANI fibers surround CNTs thoroughly. The excellent disperse has brought fine mechanical and electrical characterization for these nanocomposites.

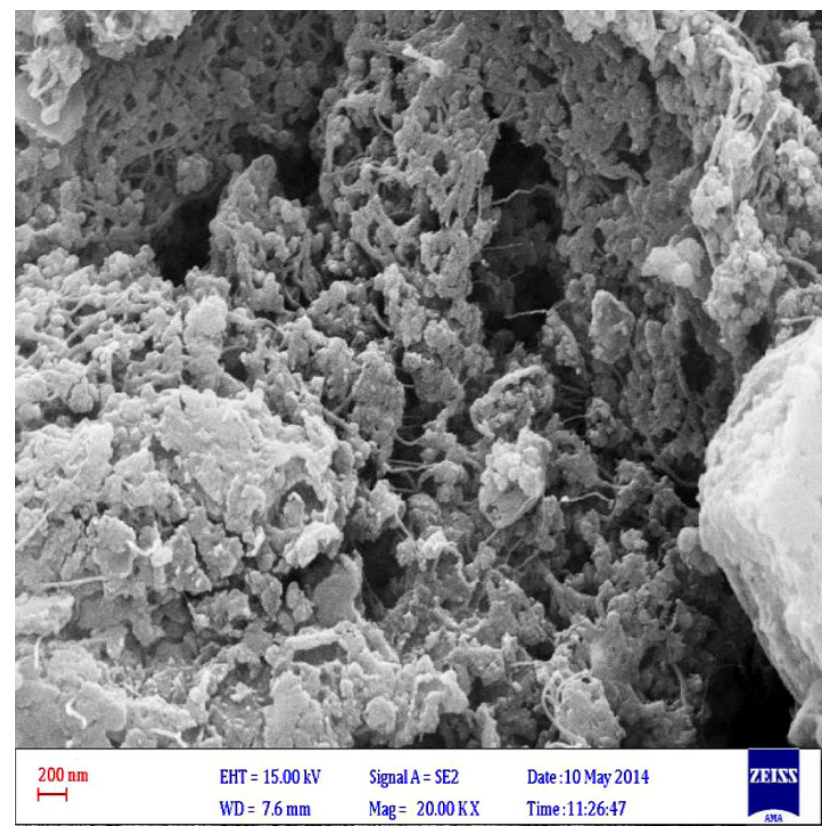

Fig. 1 SEM image of PANI/SWNT

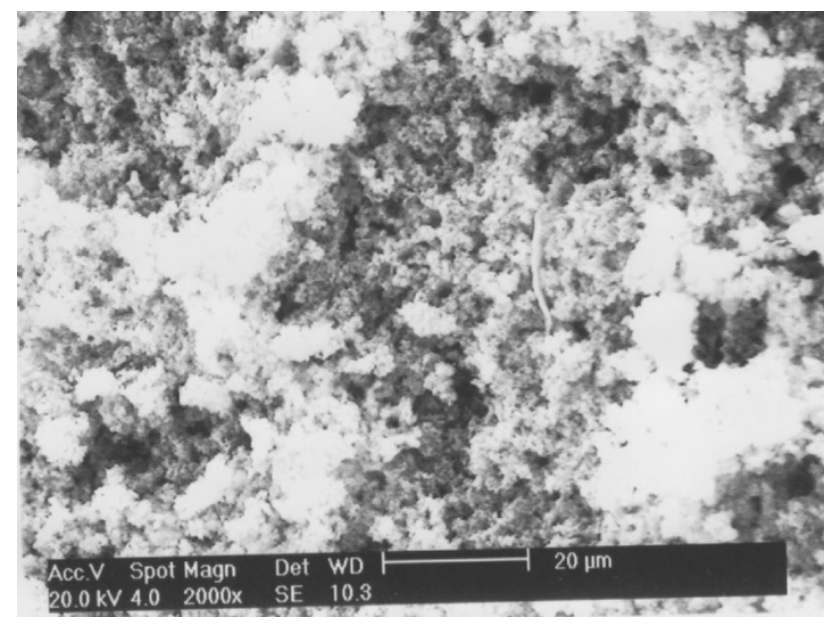

Fig. 2 SEM image of PANI/MWNT
Characterization of PANI/CNT/GNS composites

For preparation of this composite the value of CNTs should be very minor than GNS, because GNS surfaces are more than CNT surfaces, hence there exists more situation for holding aniline monomers on the carbon surface. Therefore, CNTs connected to GNS sheets covered with PANI together and arrayed the arrangement of these sheets. Figure 3 shows SEM image of PANI/GNS/CNT.

In this picture despite, existing impure compounds, but represent good dispersing of GNS and CNT in the polymer matrix.

\section{FT-IR spectroscopy}

The FT-IR spectra for the PANI are depicted in Fig. 4. The appearance of characteristic absorption band around $1,250 \mathrm{~cm}^{-1}$, which is related to the $\mathrm{C}-\mathrm{N}$ stretching in bipolar on structure, can be observed for the sample. These results indicate that polymer is highly doped and existed in conducting emeraldine salt form (ES). The broad absorption band ranges from 3,000 to $3,340 \mathrm{~cm}^{-1}$, which is attributed to the protonation of amine functional group at polymer backbone and is observed for the highly doped PANI Emeraldine salt. The absorption band near 2,908 and $2,840 \mathrm{~cm}^{-1}$ is assigned to aliphatic $\mathrm{C}-\mathrm{H}$ stretching of the polymer. The appearance of the absorption peak in the spectra is beyond our expectation as it indicates the existence of aliphatic alkyl functional group in the polymers, although none of such substances has been introduced in the system during the polymerization process. Hence, the appearance of the absorption peak may be corresponding to the long alkyl tail of the surfactant. It is well known that the surfactants can become the secondary dopants and absorbed as an outer layer surrounding the PANI particles.

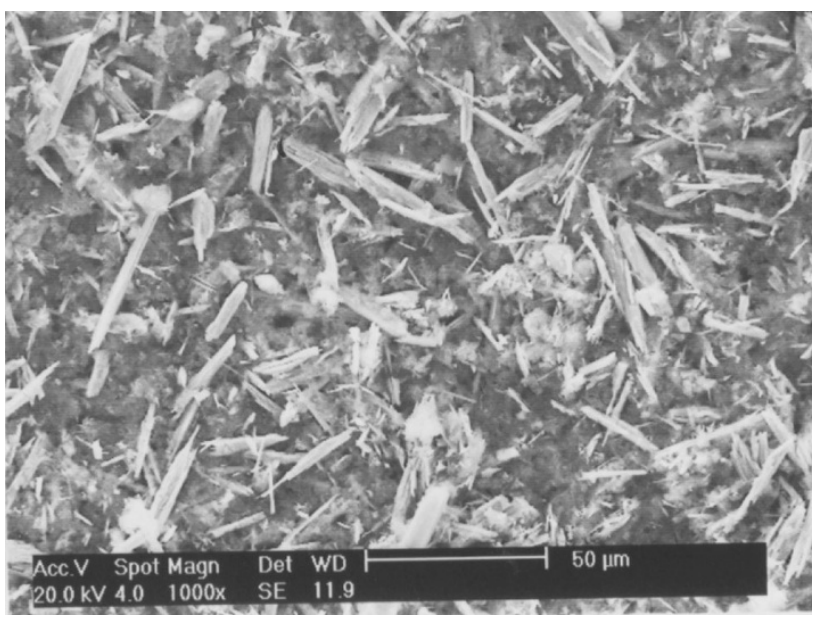

Fig. 3 SEM image of PANI/CNT/GNS 


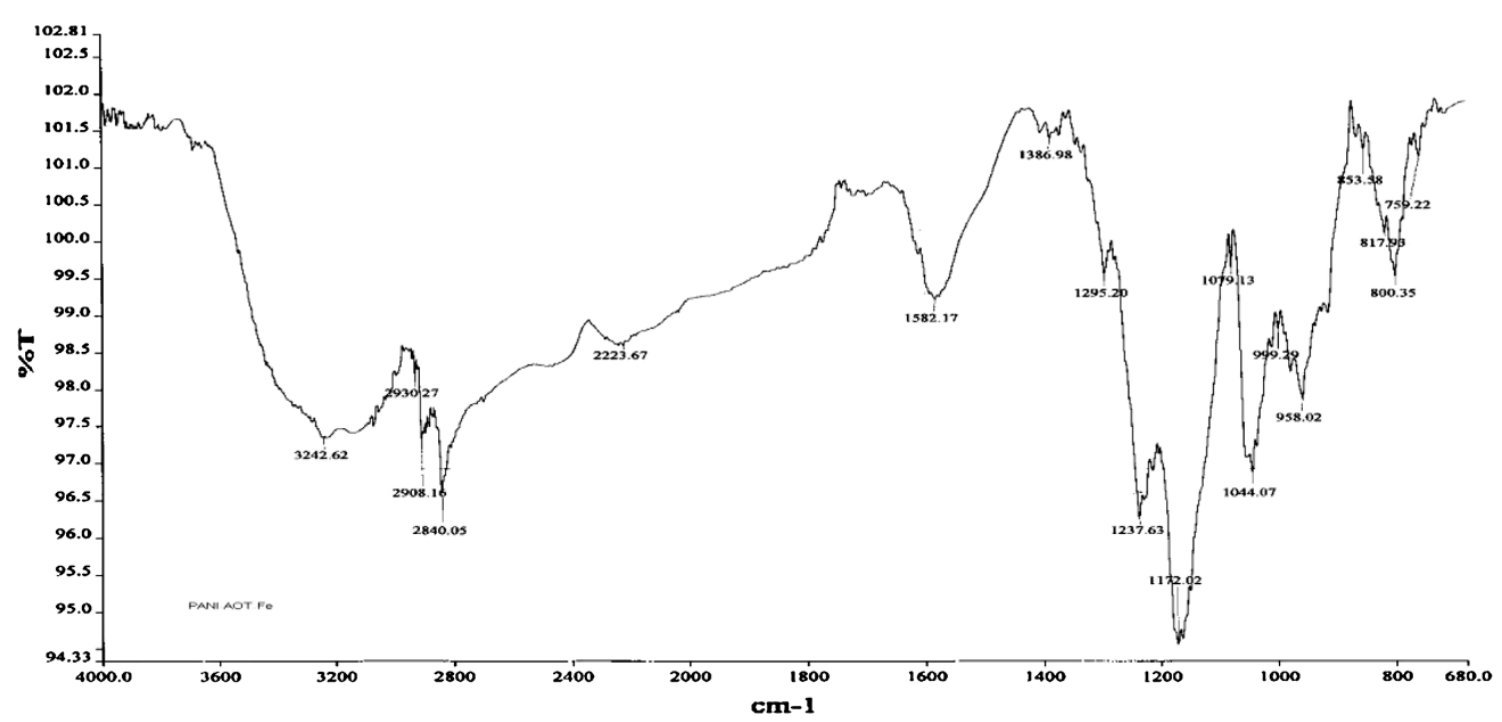

Fig. 4 FT-IR spectra of Polyaniline

\section{Conclusion}

In this paper, PANI/SWNT, PANI/MWNT and PANI/ CNT/GNS composites were synthesized using in situ polymerization. The introduction of SWNT, MWNT and GNS in these composites provides high conductivity as well as a relatively large area on which to deposit the PANI particles, which could effectively reduce the kinetic difficulties for both charge transfer and ion transport throughout the electrode. Nanoparticles that contained carboxylic acid groups were used as a core in the formation of tubular shells of PANI/nanoparticles composites. Aniline molecules were adsorbed onto, and then polymerized on the surfaces of nanoparticles. The incorporation of the nanoparticles was established with SEM technique. The in situ polymerization method can be easily applied for the construction of numerous composites with different modified nanoparticles. These composites can be used in preparation of supercapacitor electrodes.

Acknowledgments We gratefully acknowledge the support of this work by Islamic Azad University, for kindly supports.

Open Access This article is distributed under the terms of the Creative Commons Attribution License which permits any use, distribution, and reproduction in any medium, provided the original author(s) and the source are credited.

\section{References}

1. Wang, Y., Shi, Z., Huang, Y., Ma, Y., Wang, C., Chen, M., Chen, Y.: Supercapacitor devices based on graphene materials. Phys. Chem. 113, 13103 (2009)

2. Yan, J., Wei, T., Fan, Z., Qian, W., Zhang, M., Shen, X., Wei, F.: Preparation of graphene nanosheet/carbon nanotube/polyaniline composite as electrode material for supercapacitors. J. Power Sour. 195, 3041 (2010)

3. Zhang, K., Zhang, L.L., Zhao, X.S., Wu, J.: Graphene/polyaniline nanofiber composites as supercapacitor electrodes. Chem. Mater. 22, 1392 (2010)

4. Hu, Z.A., Xie, Y.L., Wang, Y.X., Mo, L.P., Yang, Y.Y., Zhang, Z.Y.: Polyaniline/ $\mathrm{SnO}_{2}$ nanocomposite for supercapacitor applications. Mater. Chem. Phys. 114, 990 (2009)

5. Snook, G.A., Kao, P., Best, A.S.: Conducting-polymer-based supercapacitor devices and electrodes. J. Power Sour. 196, 1 (2011)

6. Zhang, J., Kong, L.B., Wang, B., Luo, Y.C., Kang, L.: In-situ electrochemical polymerization of multi-walled carbon nanotube/ polyaniline composite films for electrochemical supercapacitors. Synth. Met. 159, 260 (2009)

7. Le Huy Nguyen, Nguyen, H.B.: Portable cholesterol detection with polyaniline-carbon nanotube film based interdigitated electrodes. Adv. Nat. Sci. Nanosci. Nanotechnol 3, 015004 (2012)

8. Bhadra, S., Khastgir, D., Ingha, N.K., Lee, J.H.: Progress in preparation, processing and applications of polyaniline. Prog. Polym. Sci. 34, 783 (2009)

9. Gupta, V., Miura, N.: Polyaniline/single-wall carbon nanotube (PANI/SWCNT) composites for. Electrochemica Acta 52, 1721 (2006)

10. Yan, J., Wei, T., Shao, B., Fan, Z., Qian, W., Zhang, M., Wei, F.: Preparation of a graphene nanosheet/polyaniline composite with high specific capacitance. Carbon 48, 487 (2010)

11. Green, M.J., Behabtu, N., Pasquali, M., Adams, W.W.: Nanotubes as polymers. Polymer 50, 4979 (2009)

12. Sahoo, N.G., Ran, S., Cho, J.W., Li, L., Chan, S.H.: Polymer nanocomposites based on functionalized carbon nanotubes. Prog. Polym. Sci. 35, 837 (2010)

13. Gupta, V., Miura, N.: Influence of the microstructure on the supercapacitive behavior of polyaniline/single-wall carbon nanotube composites. J. Power Sour. 157, 616 (2006)

14. Mi, H., Zhang, X., An, S., Ye, X., Yang, S.: Electrochemical/ chemical synthesis of highly-oriented single-crystal $\mathrm{ZnO}$ nanotube arrays on transparent conductive substrates. Electrochem. Commun. 9, 2859 (2007)

15. Georgakilas, V., Dallas, P., Niarchos, D., Boukos, N., Trapalis, C.: Silicone-functionalized carbon nanotubes for the .... Synth. Metal 159, 632 (2009) 
16. Huang, F., Vanhaecke, E., Chen, D.: In situ polymerization and characterizations of polyaniline on MWCNT powders and aligned MWCNT films. Catal. Today 150, 71 (2010)

17. Wu, T.M., Lin, Y.W., Liao, C.S.: Preparation and characterization of polyaniline/multi-walled carbon nanotube composites. Carbon 43, 734 (2005)

18. Mashat, L.A., Shin, K., Zadeh, K.K., Plessis, J.D., Han, S.H., Kojima, R.W., Kaner, R.B., Li, D., Gou, X., Ippolito, S.J., Wlodarski, W.: Graphene/polyaniline nanocomposite for hydrogen sensing. J. Phys. Chem. 114, 16168 (2010)

19. Stankovich, S., Dikin, D.A., Piner, R.D., Kohlhaas, K.A., Kleinhammes, A., Jia, Y., Wu, Y., Nguyen, S.T., Ruoff, R.S.:
Synthesis of graphene-based nanosheets via chemical reduction of exfoliated graphite oxide. Carbon 45, 1558 (2007)

20. Mo, Z., Zuo, D., Chen, H., Sun, Y., Zhang, P.: Synthesis of graphite nanosheets/AgCl/polypyrrole composites via two-step inverse microemulsion method. Eur. Polym. J. 43, 300 (2007)

21. Wang, D.W., Li, F., Zhao, J., Ren, W., Chen, Z.G., Tan, J., Wu, Z.S., Gentle, L., Lu, G.O., Cheng, H.M.: Fabrication of graphene/ polyaniline composite paper via in situ anodic electropolymerization for high-performance flexible electrode. ACS Nano 7, 1745 (2009) 